1 Agência Nacional de Saúde Suplementar (ANS), Diretoria de Desenvolvimento Setorial Rio de Janeiro (RJ), Brasil. juliana.pm@gmail.com

2 Fundação Oswaldo Cruz (Fiocruz), Escola Nacional de Saúde Pública Sergio Arouca (Ensp), Departamento de Administração e Planejamento em Saúde (Daps) - Rio de Janeiro (RJ), Brasil.

martins@ensp.fiocruz.br

3 Fundação Oswaldo Cruz (Fiocruz), Escola Nacional de Saúde Pública Sergio Arouca (Ensp), Departamento de Epidemiologia e Métodos Quantitativos em Saúde (DEMQS) - Rio de Janeiro (RJ), Brasil. iuri@fiocruz.br

\section{O mix público-privado e os arranjos de financiamento hospitalar no Brasil}

\author{
The public-private mix and the hospital financing arrangements in \\ Brazil
}

Juliana Pires Machado', Mônica Martins², luri da Costa Leite $\mathbf{3}$

RESUMO Com o objetivo de caracterizar a rede hospitalar brasileira quanto ao grau de compartilhamento SUS e planos de saúde, o artigo aborda a diversificação de fontes de financiamento, discutindo o tema no contexto do sistema de saúde caracterizado pelo mix público-privado desde a prestação de serviços até o pagamento e a gestão. Observou-se que a maioria dos hospitais era privado e atendia SUS e planos, com menor disponibilidade de leitos de alta complexidade no SUS. Não se identificou proporcionalidade entre oferta de leitos e cobertura da população por planos nem complementariedade SUS e planos. Os achados ajudam a delinear a oferta e os riscos à equidade no acesso, uso e qualidade do cuidado.

PALAVRAS-CHAVE Cobertura de serviços de saúde; Sistemas de saúde; Administração hospitalar; Equidade em saúde; Internação hospitalar.

ABSTRACT This paper seeks to characterize the Brazilian hospital network and its degree of sharing between the National Health System (SUS) and private health insurance. It explores the funding sources diversification and the overlapping of services, payments and management related to the public-private mix. Most hospitals were private and took both SUS and private insurance. The availability of SUS beds was lower than that of private beds. The supply of private beds was not proportional to the population covered by private insurance, and it was not complementary to the SUS beds. The findings highlight the risk to equity of access, completeness and quality of the care provided.

KEYWORDS Health services coverage; Health systems; Hospital administration; Equity in Health; Hospitalization. 


\section{Introdução}

A relação entre arranjos organizacionais, envolvendo aspectos gerenciais, financeiros e estruturais, e sua influência sobre o funcionamento e desempenho das instituições de saúde tem sido amplamente discutida. Desse debate, emergem tipologias para a classificação dos estabelecimentos e análises comparativas. A diversidade de arranjos organizacionais, especialmente em termos financeiros e gerenciais, é maior entre as instituições hospitalares (HARDING; PREKER, 2003; JAKAB ET AL., 2002; LA FORGIA; COUTTOLENE, 2009). $\mathrm{Na}$ rede de atenção à saúde brasileira, tal diversidade é visível, contudo há particularidades, sobretudo no que diz respeito às fontes de pagamento constituintes dos arranjos de financiamento dos serviços. Não é exceção pacientes utilizarem simultaneamente serviços financiados pelo Sistema Único de Saúde (SUS) e por planos privados de saúde; muitos profissionais de saúde têm mais de um emprego remunerados por essas duas fontes (VICTORA ET AL., 2011). Além disso, há instituições públicas e privadas prestando cuidado a pacientes financiados pelas duas fontes de pagamento. Embora ainda sejam raros os estudos, estão presentes na agenda sanitária preocupações com a variação no padrão de cuidado prestado aos dois grupos, com destaque aos problemas de acesso, efetividade e equidade decorrentes da fonte e valor do reembolso (VICTORA ET AL., 2011).

À complexidade do sistema de saúde, soma-se aquela concernente à gestão dos hospitais, pois nestes estão concentrados grande parte dos recursos financeiros, materiais, tecnológicos e humanos. Apesar disso, diferentemente de outros setores da economia regidos pela relação custo-volume-lucro, em geral a gestão prevalente nos hospitais é a do regime de caixa (VECINA-NETO; MALIK, 2007). Embora esta não comprometa a busca por lucratividade, aponta a complexidade da gestão financeira dessas instituições. A diversificação das fontes de financiamento tem sido adotada por instituições hospitalares no Brasil com o intuito de amenizar o risco de endividamento decorrente de eventual insuficiência em uma das fontes de pagamento (CARVAlHo, 2007). Essas estratégias de sobrevivência e expansão do espaço no mercado também influenciam o modelo de gestão. Sobressaem, assim, as tentativas de parcerias e alianças 'público-privadas', buscando relações entendidas como 'ganha-ganha' (MEDICI, 2011; MORICl; BARBOSA, 2013). Dessa forma, a iniciativa privada vem expandindo suas atividades. Nesse contexto, além de prestadores de serviços de saúde e gestores de planos de saúde, passaram a atuar também na gestão de unidades públicas - por meio de Organizações Sociais ou com a terceirização de serviços profissionais (MACHADO, 2001).

No cenário atual, os planos privados de saúde exercem forte influência e pressão sobre os hospitais de sua rede credenciada, uma vez que são importantes financiadores e reguladores do padrão de utilização com vistas à contenção de custos (VECINA-NETO; MALIK, 2007). Pressão semelhante é exercida pela administração pública sobre os hospitais públicos e privados sem fins lucrativos, por exemplo, recentemente passando a condicionar o repasse de recursos financeiros à melhoraria do desempenho, por meio das contratualizações (LIMA; RIVERA, 2012). Em paralelo, sistematicamente prestadores privados, majoritariamente sem fins lucrativos, e suas entidades de representação queixam-se dos baixos valores de reembolso predefinidos na tabela SUS, mas sobrevivem economicamente do aporte financeiro de recursos públicos, já que aproximadamente dois terços de sua clientela são de pacientes do SUS (MEDICI, 2011; VICTORA ET AL., 2011).

Com vistas a contribuir para a reflexão e a subsidiar o debate a respeito da oferta de rede compartilhada por pacientes financiados pelo SUS ou por planos de saúde, o objetivo deste estudo é caracterizar a rede hospitalar instalada no Brasil quanto aos arranjos de financiamento adotados e ao grau de compartilhamento. 


\section{Metodologia}

A principal fonte de informações foi $o$ Cadastro Nacional de Estabelecimentos de Saúde (CNES) do Ministério da Saúde. Para estimar os limites na cobertura deste cadastro, foram utilizadas como referência as informações sobre hospitais e leitos obtidos pela Pesquisa de Assistência Médico-Sanitária do Instituto Brasileiro de Geografia e Estatística (AMS/IBGE). Complementarmente, foram utilizadas as bases de dados do Registro de Planos de Saúde (RPS) da Agência Nacional de Saúde Suplementar (ANS), do Sistema de Informações Hospitalares (SIH) do SUS e da Comunicação de Informação Hospitalar (CIH) do Ministério da Saúde. Apesar do conhecido problema de baixa cobertura das informações sobre internações não SUS na base do CIH, este problema não foi considerado relevante para as análises aqui realizadas, pois a informação sobre produção hospitalar, extraída do SIH e CIH, foi somente aplicada para validar os dados cadastrais sobre tipo de convênio obtidos no CNES e RPS (MACHADO ET AL., 2013).

Para a seleção dos estabelecimentos de saúde, filtraram-se aqueles que realizavam internação e que possuíam leitos para internação no CNES. Foram incluídos os estabelecimentos ativos nesse sistema de informação, considerando sua atuação em pelo menos um ano no período entre 2008 e 2010. Neste estudo, tais estabelecimentos foram denominados 'hospitais' independentemente da nomenclatura utilizada no CNES.

O tipo de convênio informado pelos hospitais como fonte de pagamento (SUS, planos de saúde, desembolso direto ou outras fontes) foi empregado para criar a tipologia denominada 'arranjos de financiamento'. Para classificar os hospitais segundo os arranjos de financiamento, utilizou-se a informação de convênio do CNES e sua vinculação a alguma operadora de plano de saúde no RPS da ANS. Sequencialmente, a informação sobre volume de internação por hospital no SIH e CIH foi utilizada para detectar as internações por fontes de pagamento não registradas nos cadastros do CNES e RPS. O número de registro do hospital no CNES foi a variável-chave para criação dessa classificação. Assim, três categorias de arranjos de financiamento foram formadas: 'Somente SUS', 'Planos e particular' e misto -'SUS, planos e particular'. Arranjo 'Somente SUS' incluiu os hospitais sem convênio com planos no CNES e sem internações privadas registradas na CIH. Arranjo 'Planos e particular' incluiu hospitais conveniados a planos, sem convênio com SUS no CNES e sem internações SUS informadas no SIH. Arranjo 'SUS, planos e particular' (clientela mista) incluiu os hospitais conveniados a planos e ao SUS no CNES, e/ou com internações reembolsadas por ambas as fontes registradas no $\mathrm{SIH}$ e $\mathrm{CIH}$.

Para a análise dos dados, comparou-se a distribuição dos hospitais por fontes de pagamento e arranjos de financiamento com a cobertura da população por planos de saúde. A informação sobre a população coberta por planos de saúde é oriunda do Sistema de Informações de Beneficiários (SIB) da ANS.

$\mathrm{O}$ cálculo da oferta de leitos destinados à população beneficiária de planos de saúde foi realizado utilizando no numerador o número de leitos não SUS e no denominador o número de beneficiários. A disponibilidade de leitos para a população usuária exclusiva do SUS foi calculada utilizando no numerador o número de leitos SUS e no denominador a diferença entre a população total e os beneficiários de planos de saúde.

A complexidade da estrutura dos hospitais nos estados e regiões brasileiras foi explorada com base na existência e proporção de leitos de Unidade de Terapia Intensiva (UTI) dos tipos II ou III. A classificação dos tipos de UTI é descrita na Portaria do Ministério da Saúde GM no 3.432/1998 (BRASIL, 1998). A informação sobre os leitos de UTI do tipo I foi excluída, pois essa categoria não refere 
os requisitos mínimos de disponibilidade de equipe, serviços, exames, equipamentos e materiais exigidos na mesma portaria ministerial. Na ausência de um parâmetro mais preciso, para o cálculo dos valores mínimo e máximo de leitos de UTI considerados necessários, aplicou-se o percentual definido pelo Ministério da Saúde na Portaria GM n ${ }^{\circ}$ $1.101 / 2002$ (BRASIL, 2006), que estabelece os parâmetros assistenciais para o SUS com base em dados de produção.

Todas as informações aqui utilizadas são de domínio público e acesso livre. Contudo, este trabalho faz parte do estudo 'O arranjo público-privado e a qualidade da assistência hospitalar no Brasil', aprovado pelo Comitê de Ética da Escola Nacional de Saúde Pública (CAAE número 02234312.3.0000.5240) em 21 de agosto de 2012.

\section{Resultados}

A análise preliminar para estimar os limites na cobertura dos dados indicou um número de hospitais cadastrados no CNES em 2009 próximo daquele registrado pela AMS, em todas as regiões do País (compatibilidade de cerca de 100,0\%), incluindo a distribuição segundo natureza pública ou privada e vínculo com o SUS. Quanto aos leitos existentes, estes eram aproximadamente 12\% maior no CNES quando comparado à AMS.

Dos 7.161 hospitais cadastrados no CNES, 40,6\% (2.905) eram públicos, 34,7\% (2.483) privados com fins lucrativos e $24,7 \%$ (1.773) privados sem fins lucrativos. Cerca de $46,6 \%$ dos hospitais (3.336) atendiam a clientela mista, com arranjo de financiamento 'SUS, planos e particular' (tabela 1).

Tabela 1. Hospitais, leitos e internações segundo arranjos de financiamento - Brasil, 2008 a 2010

\begin{tabular}{|c|c|c|c|c|c|c|c|c|}
\hline \multirow{2}{*}{$\begin{array}{l}\text { Natureza Jurídica / } \\
\text { Fonte de Pagamento }\end{array}$} & \multicolumn{2}{|c|}{ Planos e Particular } & \multicolumn{2}{|c|}{ Somente SUS } & \multicolumn{2}{|c|}{ SUS, Planos e Particular } & \multicolumn{2}{|c|}{ Total } \\
\hline & (n) & $(\%)$ & (n) & $(\%)$ & (n) & $(\%)$ & (n) & $(\%)$ \\
\hline Hospitais & 1.383 & 19,3 & 2.442 & 34,1 & 3.336 & 46,6 & 7.161 & 100,0 \\
\hline Público & 39 & 1,3 & 2.217 & 76,3 & 649 & 22,3 & 2.905 & 100,0 \\
\hline Privado Sem Fins Lucrativos & 72 & 4,1 & 144 & 8,1 & 1.557 & 87,8 & 1.773 & 100,0 \\
\hline Privado Com Fins Lucrativos & 1.272 & 51,2 & 81 & 3,3 & 1.130 & 45,5 & 2.483 & 100,0 \\
\hline Leitos Existentes & 70.277 & 14,1 & 112.947 & 22,6 & 315.970 & 63,3 & 499.194 & 100,0 \\
\hline Público & 2.639 & 1,5 & 99.988 & 55,3 & 78.339 & 43,3 & 180.966 & 100,0 \\
\hline Privado Sem Fins Lucrativos & 7.739 & 4,5 & 7.102 & 4,1 & 156.601 & 91,3 & 171.442 & 100,0 \\
\hline Privado Com Fins Lucrativos & 59.899 & 40,8 & 5.857 & 4,0 & 81.030 & 55,2 & 146.786 & 100,0 \\
\hline Leitos SUS & 92 & 0,0 & 110.519 & 31,1 & 244.618 & 68,9 & 355.229 & 100,0 \\
\hline Público & 10 & 0,0 & 98.436 & 56,8 & 74.861 & 43,2 & 173.307 & 100,0 \\
\hline Privado Sem Fins Lucrativos & 82 & 0,1 & 6.752 & 5,5 & 116.774 & 94,5 & 123.608 & 100,0 \\
\hline Privado Com Fins Lucrativos & 0 & 0,0 & 5.331 & 9,1 & 52.983 & 90,9 & 58.314 & 100,0 \\
\hline Leitos Não SUS & 70.185 & 48,8 & 2.428 & 1,7 & 71.352 & 49,6 & 143.965 & 100,0 \\
\hline Público & 2.629 & 34,3 & 1.552 & 20,3 & 3.478 & 45,4 & 7.659 & 100,0 \\
\hline Privado Sem Fins Lucrativos & 7.657 & 16,0 & 350 & 0,7 & 39.827 & 83,3 & 47.834 & 100,0 \\
\hline Privado Com Fins Lucrativos & 59.899 & 67,7 & 526 & 0,6 & 28.047 & 31,7 & 88.472 & 100,0 \\
\hline Internações 2008-2010 & 2.844 .519 & 7,3 & 8.599 .358 & 22,1 & 27.463 .940 & 70,6 & 38.907 .817 & 100,0 \\
\hline SUS & 0 & 0,0 & 8.599 .358 & 26,3 & 24.120 .116 & 73,7 & 32.719 .474 & 100,0 \\
\hline Não SUS & 2.844 .519 & 46,0 & 0 & 0,0 & 3.343 .824 & 54,0 & 6.188 .343 & 100,0 \\
\hline
\end{tabular}

Fonte: Ministério da Saúde. Cadastro Nacional de Estabelecimentos de Saúde (CNES), Registro de Planos de Saúde (RPS), Sistema de Informações Hospitalares do SUS $(\mathrm{SIH})$ e Comunicação de Internação Hospitalar $(\mathrm{CIH})$. 
Os hospitais públicos majoritariamente atendiam exclusivamente ao SUS, mas 649 (22,3\%) também informaram possuir convênio com planos de saúde e receber pagamento particular. Os privados sem fins lucrativos eram majoritariamente mistos, contabilizando 1.557 hospitais (87,8\%) com arranjo 'SUS, planos e particular'. Já os privados com fins lucrativos dividiam-se em 1.272 (51,2\%) com arranjo 'Planos e particular' e 1.130 (45,5\%) com arranjo misto: 'SUS, planos e particular' (tabela 1).

Dos 499.194 leitos existentes no País no período de análise, 315.970 (63,3\%) estavam em hospitais de arranjo misto (SUS, planos e particular) e 112.947 (22,6\%) em hospitais de arranjo 'Somente SUS', resultando em 355.229 leitos disponíveis para o SUS $(71,2 \%$ do total). Já os leitos não SUS disponíveis nos hospitais de arranjo 'Planos e particular' somavam 70.277 (14,1\% do total) (tabela 1).

No período de 2008 a 2010, os estabelecimentos de arranjo misto foram responsáveis pela maior parte das internações informadas do País (70,6\%), tanto as pagas pelo SUS $(73,7 \%)$ como as pagas por plano de saúde ou particular $(54,0 \%)$. Os hospitais 'Somente SUS' realizaram 22,1\% de todas as internações e $26,3 \%$ das internações pelo SUS. Já os hospitais de arranjo 'Planos e particular' realizaram 7,3\% das internações do País e 46,0\% das internações não SUS (tabela 1).

Quanto ao porte, 5.313 hospitais possuíam até 79 leitos (74,2\%), 393 deles possuíam entre 80 e 99 leitos (5,5\%), 959 entre 100 e 199 leitos (13,4\%) e 496 tinham 200 ou mais leitos (6,9\%). O arranjo 'Somente SUS' apresentou a maior proporção de hospitais de menor porte, $86,9 \%$ destes hospitais possuíam menos de 80 leitos. Os hospitais de arranjo misto (SUS, planos e particular) apresentaram a maior proporção $(10,9 \%)$ de unidades de maior porte maior a 199 leitos (gráfico 1).

Gráfico 1. Hospitais segundo o número de leitos por arranjos de financiamento - Brasil, 2008 a 2010.

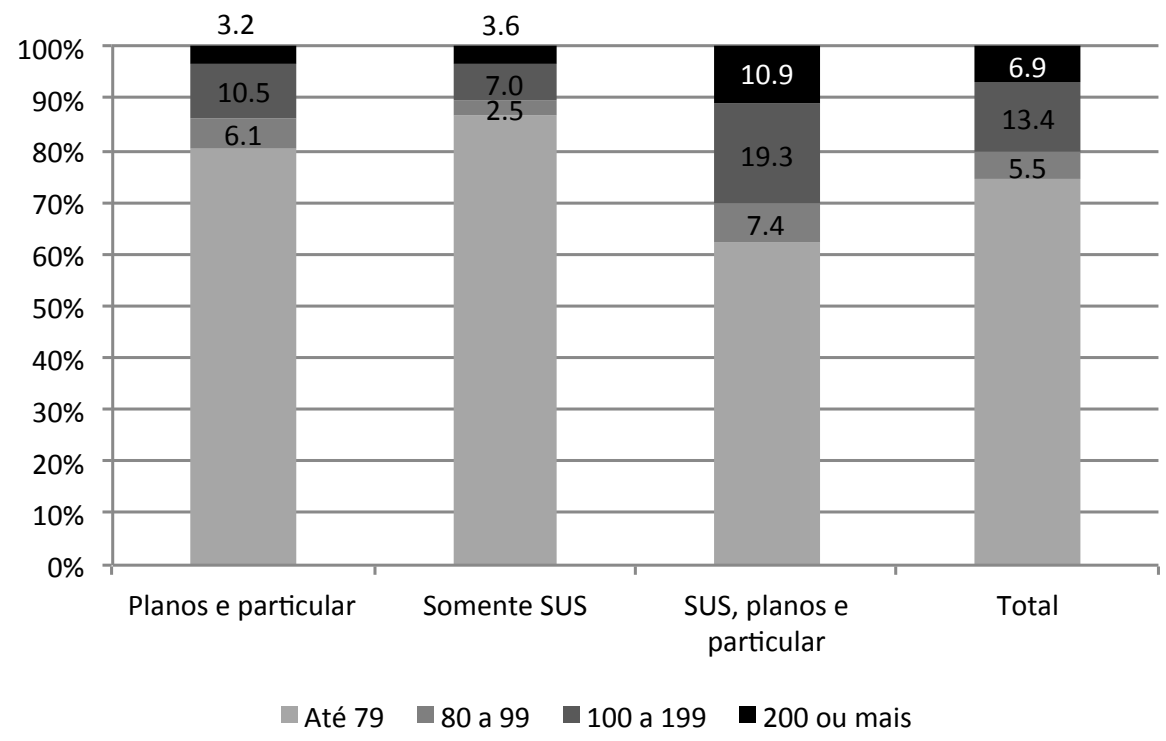


A maioria dos estabelecimentos com arranjo 'Planos e particular' encontrava-se em São Paulo, Rio de Janeiro, Minas Gerais e Goiás. Já aqueles com arranjo 'Somente
SUS' estavam em maior número na Bahia, Pernambuco, Maranhão e São Paulo. Os hospitais com arranjo misto (SUS, planos e particular) localizavam-se majoritariamente

Tabela 2. Hospitais por arranjo de financiamento e disponibilidade de leitos SUS e não SUS segundo região e UF - Brasil, 2008 a 2010

\begin{tabular}{|c|c|c|c|c|c|c|c|c|c|c|}
\hline \multirow[t]{2}{*}{ Regiões e Estados } & \multicolumn{4}{|c|}{ Estabelecimentos por Arranjos } & \multicolumn{3}{|c|}{ Leitos } & \multicolumn{3}{|c|}{ Leitos por mil hab. } \\
\hline & Total & $\begin{array}{l}\text { Planos e } \\
\text { Particular }\end{array}$ & $\begin{array}{l}\text { Somente } \\
\text { SUS }\end{array}$ & $\begin{array}{l}\text { SUS, Planos e } \\
\text { Particular }\end{array}$ & Total & SUS & Não SUS (1) & Total & SUS (2) & Não SUS (3) \\
\hline Brasil & 7.161 & 1.383 & 2.442 & 3.336 & 499.194 & 355.229 & 143.965 & 2,6 & 2,4 & 3,4 \\
\hline Região Norte & 634 & 118 & 328 & 188 & 34.333 & 26.228 & 8.105 & 2,2 & 1,9 & 5,7 \\
\hline Rondônia & 104 & 36 & 54 & 14 & 4.319 & 3.232 & 1.087 & 2,9 & 2,4 & 8,5 \\
\hline Acre & 31 & 3 & 16 & 12 & 1.549 & 1.403 & 146 & 2,2 & 2,2 & 3,4 \\
\hline Amazonas & 117 & 18 & 69 & 30 & 7.064 & 5.775 & 1.289 & 2,1 & 1,9 & 3,2 \\
\hline Roraima & 17 & 2 & 6 & 9 & 846 & 795 & 51 & 2,0 & 2,0 & 2,0 \\
\hline Pará & 279 & 43 & 131 & 105 & 16.658 & 11.675 & 4.983 & 2,2 & 1,7 & 7,3 \\
\hline Amapá & 24 & 7 & 11 & 6 & 1.264 & 1.056 & 208 & 2,0 & 1,9 & 3,3 \\
\hline Tocantins & 62 & 9 & 41 & 12 & 2.633 & 2.292 & 341 & 2,0 & 1,9 & 5,0 \\
\hline Região Nordeste & 2.299 & 255 & 1.299 & 745 & 126.480 & 104.421 & 22.059 & 2,4 & 2,2 & 4,1 \\
\hline Maranhão & 267 & 26 & 188 & 53 & 14.609 & 12.893 & 1.716 & 2,3 & 2,1 & 5,7 \\
\hline Piauí & 210 & 15 & 113 & 82 & 8.427 & 7.321 & 1.106 & 2,7 & 2,5 & 5,5 \\
\hline Ceará & 324 & 46 & 141 & 137 & 19.960 & 15.475 & 4.485 & 2,3 & 2,0 & 4,8 \\
\hline Rio Grande do Norte & 200 & 9 & 136 & 55 & 8.030 & 6.745 & 1.285 & 2,6 & 2,5 & 2,8 \\
\hline Paraíba & 203 & 17 & 111 & 75 & 10.773 & 9.060 & 1.713 & 2,9 & 2,6 & 5,3 \\
\hline Pernambuco & 351 & 50 & 195 & 106 & 23.719 & 18.824 & 4.895 & 2,7 & 2,5 & 3,9 \\
\hline Alagoas & 98 & 8 & 53 & 37 & 6.565 & 5.571 & 994 & 2,1 & 1,9 & 3,4 \\
\hline Sergipe & 60 & 7 & 34 & 19 & 4.015 & 3.143 & 872 & 2,0 & 1,8 & 3,8 \\
\hline Bahia & 586 & 77 & 328 & 181 & 30.382 & 25.389 & 4.993 & 2,1 & 1,9 & 3,6 \\
\hline Região Sudeste & 2.319 & 687 & 443 & 1.189 & 217.390 & 139.733 & 77.657 & 2,7 & 2,6 & 2,8 \\
\hline Minas Gerais & 666 & 102 & 102 & 462 & 47.116 & 34.463 & 12.653 & 2,4 & 2,2 & 2,9 \\
\hline Espírito Santo & 122 & 32 & 23 & 67 & 8.710 & 6.202 & 2.508 & 2,5 & 2,5 & 2,4 \\
\hline Rio de Janeiro & 567 & 250 & 142 & 175 & 54.047 & 32.605 & 21.442 & 3,4 & 3,1 & 4,0 \\
\hline São Paulo & 964 & 303 & 176 & 485 & 107.517 & 66.463 & 41.054 & 2,6 & 2,7 & 2,5 \\
\hline Região Sul & 1.110 & 130 & 124 & 856 & 80.896 & 57.686 & 23.210 & 2,9 & 2,7 & 3,9 \\
\hline Paraná & 515 & 67 & 102 & 346 & 30.822 & 22.288 & 8.534 & 2,9 & 2,6 & 3,8 \\
\hline Santa Catarina & 227 & 33 & 7 & 187 & 16.160 & 11.942 & 4.218 & 2,6 & 2,5 & 3,0 \\
\hline Rio Grande do Sul & 368 & 30 & 15 & 323 & 33.914 & 23.456 & 10.458 & 3,1 & 2,7 & 4,5 \\
\hline Região Centro Oeste & 799 & 193 & 248 & 358 & 40.095 & 27.161 & 12.934 & 2,9 & 2,3 & 6,3 \\
\hline Mato Grosso do Sul & 128 & 30 & 30 & 68 & 6.220 & 4.199 & 2.021 & 2,6 & 2,1 & 5,5 \\
\hline Mato Grosso & 170 & 38 & 45 & 87 & 7.744 & 5.445 & 2.299 & 2,6 & 2,0 & 7,0 \\
\hline Goiás & 432 & 91 & 164 & 177 & 18.584 & 12.504 & 6.080 & 3,1 & 2,4 & 8,4 \\
\hline Distrito Federal & 69 & 34 & 9 & 26 & 7.547 & 5.013 & 2.534 & 2,9 & 2,6 & 3,9 \\
\hline
\end{tabular}

Fonte: Ministério da Saúde. Cadastro Nacional de Estabelecimentos de Saúde (CNES), Sistema de Informações de Beneficiários de Planos de Saúde (SIB) e Datasus.

Notas: (1) Calculado a partir da diferença entre total de leitos existentes e leitos SUS;

(2) Calculado utilizando-se a diferença entre a população e o número de beneficiários de planos privados de saúde;

(3) Calculado utilizando-se o número de beneficiários de planos privados de saúde. 
em São Paulo, Minas Gerais, Paraná e Rio Grande do Sul. Nos estados das regiões Sul e Sudeste, observou-se uma forte concentração de hospitais conveniados a planos de saúde (arranjos 'Planos e particular' e 'SUS, planos e particular') (tabela 2).

A razão entre número de leitos e população mostrou que havia 2,6 leitos para cada mil habitantes no Brasil. O estado do Rio de Janeiro apresentou a maior razão de leitos (3,4 por mil habitantes), seguido por Goiás, Rio Grande do Sul, Distrito Federal, Rondônia, Paraná e Paraíba, cujas razões foram maiores do que a razão nacional. Por outro lado, os estados de Tocantins, Amapá, Roraima e Sergipe apresentaram as menores razões de leitos, 2,0 por mil habitantes (tabela 2).

A oferta de leitos foi maior para a população beneficiaria de planos de saúde (3,4 leitos por mil beneficiários de planos privados de saúde) quando comparada à população usuária exclusivamente do SUS (2,4 leitos por mil usuários). No entanto, houve menor variabilidade regional na oferta dos leitos para pacientes do SUS em relação aos beneficiários de planos de saúde: enquanto a razão de leitos entre usuários do SUS variou de 1,7 (no Pará) a 3,1 (no Rio de Janeiro), entre beneficiários de planos de saúde esta razão variou de 2,0 (em Roraima) a 8,5 (em Rondônia). As maiores diferenças na oferta de leitos SUS e não SUS foram observadas nos estados de Mato Grosso, Pará, Goiás e Rondônia, onde os leitos por beneficiários de planos superavam em mais de três vezes os leitos por usuários do SUS. Vale ressaltar que, apenas nos estados de São Paulo e Espírito Santo, a disponibilidade de leitos SUS foi maior do que a de leitos não SUS (tabela 2).

A relação entre cobertura por planos privados de saúde e oferta de leitos não SUS não apresentou proporcionalidade. Os estados da região Sudeste, além do Distrito Federal e Santa Catarina, destacaram-se pela maior cobertura da população por planos, sem maior disponibilidade de leitos (gráfico 2).

Gráfico 2. Leitos não SUS por mil beneficiários de planos de saúde e cobertura da população por planos de saúde - estados do Brasil, 2008 a 2010

— Leitos não SUS/1.000 beneficiários —Cobertura por planos (\%)

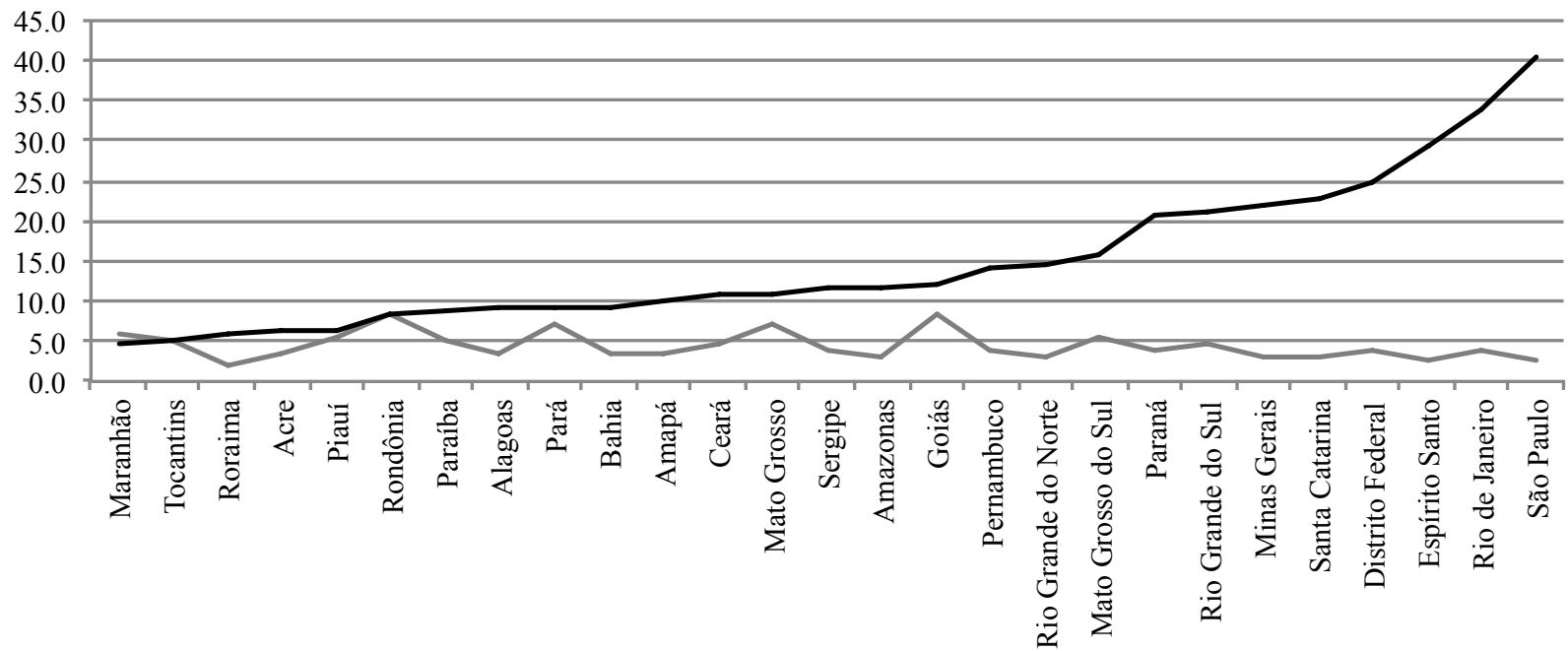

Fonte: Ministério da Saúde. Cadastro Nacional de Estabelecimentos de Saúde (CNES), Registro de Planos de Saúde (RPS) e Sistema de Informações de Beneficiários de Planos de Saúde (SIB). 
Os 24.475 leitos de UTI existentes encontravam-se localizados em apenas 1.205 hospitais do País, dos quais a maioria (72,9\%) tinha arranjo 'SUS, planos e particular'. Dos hospitais com UTI, 46\% (559 hospitais) encontravam-se na região Sudeste, principalmente no estado de São Paulo. Em todos os estados brasileiros, a disponibilidade de leitos de UTI foi maior para a população usuária de planos de saúde do que para aquela usuária exclusiva do SUS. A única exceção foi o estado de São Paulo, onde a disponibilidade de leitos de
UTI para a população usuária exclusiva do SUS superava aquela para a população não usuária exclusiva SUS. O gráfico 3 consolida as informações sobre leitos de UTI por região e arranjo de financiamento. A região Sudeste apresentou as menores discrepâncias entre leitos de UTI disponíveis para as duas populações. O Brasil alcançou, na média, o mínimo de leitos de UTI estimados pelo Ministério da Saúde (4\% do total de leitos existentes). No entanto, as regiões Norte e Nordeste não atingiram este patamar mínimo (gráfico 3).

Gráfico 3. Faixas mínima e máxima de leitos de UTI recomendada* e leitos de UTI existentes por 10 mil habitantes, segundo arranjos de financiamento dos estabelecimentos - regiões do Brasil, 2008 a 2010

- - Mínimo (4\% dos leitos) - - - Máximo (10\% dos leitos)

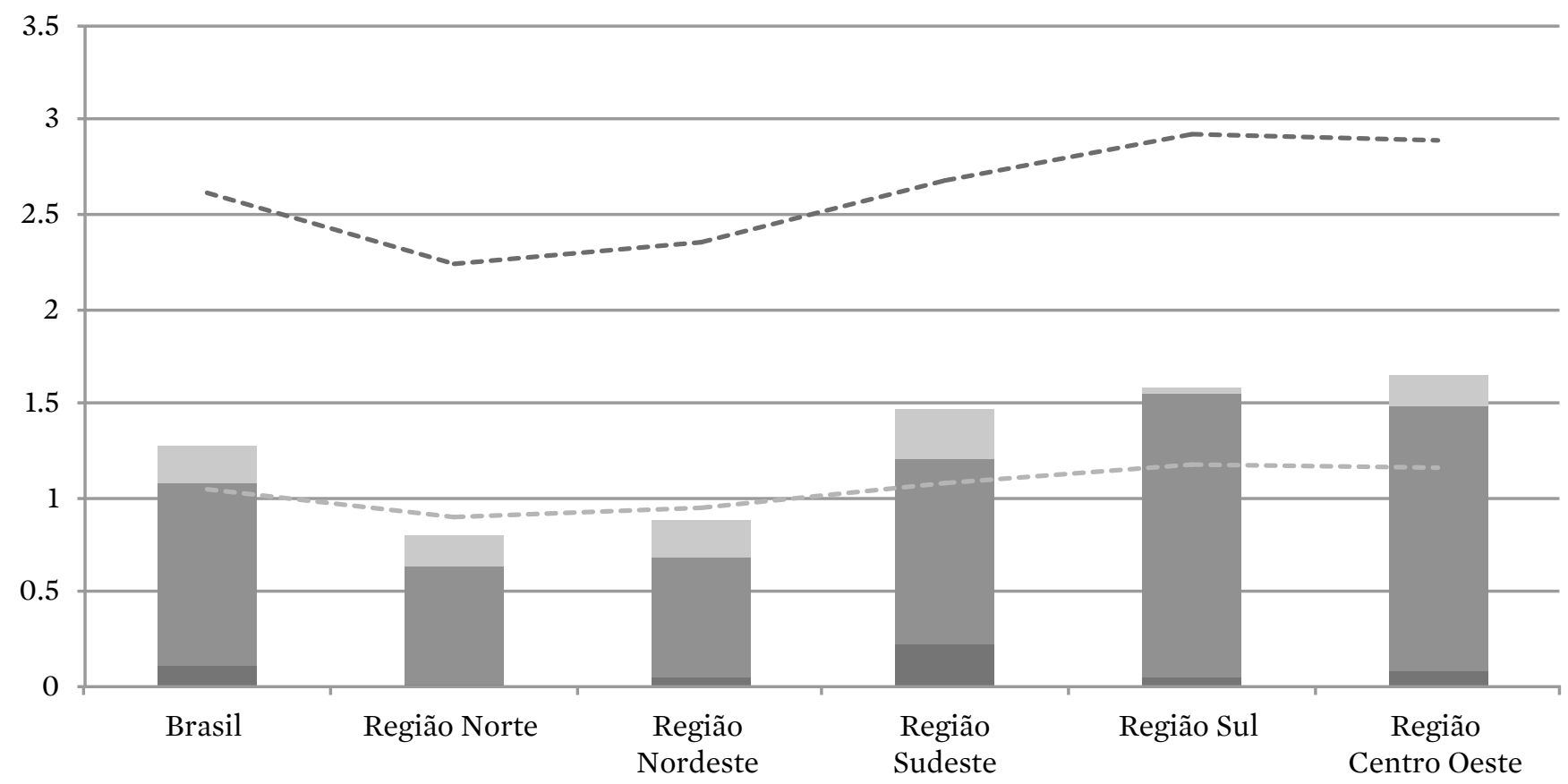

Fonte: Ministério da Saúde. Cadastro Nacional de Estabelecimentos de Saúde (CNES), Sistema de Informações Hospitalares do SUS (SIH), Comunicação de Informação Hospitalar (CIH), Sistema de Informações de Beneficiários de Planos de Saúde (SIB), Registro de Planos de Saúde (RPS).

Nota: * Parâmetro recomendado pela Portaria ministerial no. 1.101/2002.

\section{Discussão}

A diversificação das fontes de financiamento adotadas por hospitais, independentemente da natureza jurídica, contribui para que as fronteiras entre o público e o privado no sistema de saúde brasileiro se tornem cada vez menos demarcadas, com uma rede 
majoritariamente mista e partilhada. A superposição de clientelas não é exclusiva ao sistema de saúde brasileiro, mas reforça a necessidade de articulação entre os segmentos público e privado (BASU ET AL., 2012; FERRI-DEBARROS; GIBSON; HOWARD, 2012; SLOAN ET AL., 2001).

Mesmo com uma rede majoritariamente mista, tem-se no Brasil uma segmentação interna ao grupo de beneficiários de planos de saúde, em que parte deles acessa hospitais exclusivamente privados, mas a maioria conta com os serviços de hospitais privados conveniados ao SUS, compartilhando a mesma rede que atende os pacientes do SUS. Esse cenário configura três distintos perfis de acesso e utilização de serviços hospitalares, ilustrados nos dados analisados: aqueles que usam exclusivamente os serviços do SUS, os que usam planos com cobertura pelos mesmos serviços do SUS e os que usam planos com redes de serviços exclusivamente privadas.

Apesar da sobreposição das redes pública e privada, configurada pela alta ocorrência de hospitais com arranjo de financiamento misto, observou-se diferença na disponibilidade de leitos, especialmente daqueles de UTI para beneficiários de planos privados de saúde e usuários exclusivos do SUS, com vantagem para a população não SUS. Esse achado exemplifica as iniquidades que Santos (2011) apontou como derivadas da duplicação de cobertura observada no Brasil, em que há beneficiários de planos que também utilizam o SUS. Além disso, observou-se simultaneamente menor variabilidade na oferta de leitos disponíveis para o SUS em todo o País. Contudo, há importantes variações regionais na oferta de leitos disponíveis à população não SUS, indicando a falta de articulação com a cobertura por planos, bem como a não complementariedade entre os segmentos público e privado, o que seria o desejado em um sistema que propõe alcançar cobertura universal (OECD, 2004). A disponibilidade e a distribuição dos leitos de UTI para as populações SUS e não
SUS, assim como a proporção de hospitais de menor porte e menor complexidade no âmbito do SUS, indicam heterogeneidade ainda mais importante para casos de mais alta complexidade.

A importância do setor privado na atenção hospitalar e sua desarticulação com a distribuição do setor público observada neste estudo corroboram a discussão colocada por Baptista (2010) e Scheffer e Bahia (2013). Estes autores associam alguns desses problemas à não explicitação das prioridades estatais para a conformação do sistema de saúde no Brasil. Mais especificamente discutindo o papel do setor suplementar de saúde, Ferri-de-Barros et al. (2012) apontam a falta de orientação política formal nesta área e desarticulação desta com aquelas definidas no âmbito do SUS, em sintonia com os achados que apontaram a instalação desordenada de leitos, não respeitando a cobertura por planos de saúde ou a instalação de leitos SUS.

Nesse contexto, a natureza jurídica dos estabelecimentos é insuficiente para indicar distinções de clientela SUS ou não SUS. Regionalmente, foi possível confirmar a importância de hospitais de natureza pública exclusivos para pacientes do SUS, nas regiões Norte e Nordeste, e dos hospitais de natureza privada exclusivos para pacientes não SUS, no Sudeste. Esses achados coadunam-se às preocupações de Dain (2007) e de Santos e Gerschman (2004) a respeito da determinação econômica e política da organização de serviços entre provedores públicos e privados. Nesse sentido, a menor dependência do SUS da oferta de serviços hospitalares privados é menor no Norte e Nordeste, onde ao menos metade dos leitos disponíveis estavam em hospitais públicos. Tal fato indica que os investimentos governamentais em termos de oferta hospitalar privilegiaram áreas geográficas com menor penetração de empresas privadas de saúde, hospitais e também planos de saúde, quadro esse que pode suscitar interpretações positivas ou negativas, 
mas sobretudo debates importantes entre diferentes atores.

Nesse âmbito, é necessária a reflexão sobre questões centrais relacionadas com a regulação do sistema de saúde, tais como: os mecanismos de pagamento; as melhores opções de parceria público-privada; os dispositivos e diretrizes da regulação do Estado sobre o setor e a capacidade de sustentação do modelo de prestação de serviços existente (LA FORGIA; COUTTOLENE, 2009; VECINA-NETO; MALIK, 2007).

Destacam-se como principais limites deste estudo a qualidade e completitude de informações das bases de dados. Isto impede o conhecimento pleno do volume de internações realizadas no País, influenciando a construção de cenários úteis para a gestão em saúde, bem como a problematização relacionada com a atuação de alguns hospitais nas duas frentes (SUS e não SUS) e com a dualidade do sistema público-privado instalado no País.

Ainda assim, os achados deste estudo podem contribuir especialmente no âmbito da organização da rede prestadora de serviços hospitalares brasileira. Além da oferta dos recursos, também devem ser considerados os riscos ao acesso, a adequação e a efetividade dos cuidados prestados, bem como sua equidade. Dessa forma, é importante que a tomada de decisão seja consciente do seu papel no delineamento do sistema de saúde do País, podendo contribuir para a legitimação do sistema universal proposto em nossa Constituição Federal, ou para afastar-se dele.

\section{Considerações finais}

A tendência contemporânea de busca por novas formas de financiamento, com a adoção de múltiplas fontes de pagamento, mostrou-se real para a rede hospitalar brasileira, conformando um cenário caracterizado pela dupla porta de entrada: via SUS ou planos de saúde. Além disso, as desigualdades na oferta de leitos não SUS e a não complementariedade com a oferta de leitos SUS indicam que esta rede não se distribui com a missão principal de atender à demanda e que, além disso, não parece haver coordenação entre os segmentos público e privado. Ainda que a maior concentração de leitos para internação esteja nas regiões mais ricas, onde também se encontra a maior parte dos beneficiários de planos de saúde, certamente a desigualdade de distribuição dos serviços e a não complementariedade dos segmentos público e privado afetam a oferta, que por sua vez afeta o acesso aos serviços de saúde.

Esforços devem ser feitos no sentido de tornar o acesso à rede hospitalar mais equânime no País, independentemente da fonte de pagamento usada pelos pacientes. No âmbito do SUS, o aumento do número de leitos e de leitos de UTI por habitantes parece essencial para aumentar o acesso ao cuidado de maior complexidade. No âmbito privado, a regulação da implantação de novos serviços e do acesso aos serviços existentes via planos privados de saúde deve ser colocada em prática, a exemplo de outros países. Diante da rede sobreposta e heterogênea existente, esta regulação é fundamental para evitar 'leilões' dos serviços existentes entre os beneficiários de planos privados, com a venda de promessas de acesso ao cuidado de melhor qualidade que possivelmente não se concretizarão.

Além do alinhamento de esforços públicos e privados com as necessidades de serviços hospitalares da população, o monitoramento regular da qualidade destes serviços deve fazer parte do arcabouço de informações usadas no direcionamento de políticas e regulamentações na área da atenção hospitalar no Brasil, como elementos vitais para aproximar o planejamento e o cuidado em saúde das discussões teóricas e científicas sobre a organização do sistema e sobre a qualidade da assistência, em prol de ganhos para a sociedade e para o País. 


\section{Referências}

BAPTISTA, T. W. F. Análise da produção legislativa em saúde no Congresso Nacional brasileiro (1990-2006). Cad. Saúde Pública, Rio de Janeiro, v. 26, n. 1, p. 97-109, 2010.

BASU, S. et al. Comparative performance of private and public healthcare systems in low and middle-income countries: a systematic review. PLoS Med. on line, v. 9, n. 6, 2012. Disponível em: <http://journals. plos.org/plosmedicine/article?id=10.1371/journal. pmed.1001244>. Acesso em: 23 out. 2015.

BRASIL. Ministério da Saúde. Portaria GM/MS n. 1101, de 12 de junho de 2002. Estabelece os parâmetros de cobertura assistencial no âmbito do Sistema Único de Saúde - SUS. Diário Oficial [da] União. Brasília, DF: Ministério da Saúde,12 jul. 2006. Disponível em: <http://wwwl.saude.ba.gov.br/regulasaude/2009/ PN\%20PORTARIAS\%202009/nvos\%20pdfs\%202009/ PT\%20GM\%201101\%2012.06.2002.pdf>. Acesso em: 23 out. 2015.

Ministério da Saúde. Portaria GM/MS n. 3432, de 12 de agosto de 1998. Estabelece critérios para credenciamento de Unidades de Terapia Intensiva. Diário Oficial [da] União. Brasília, DF: Ministério da Saúde, 12 ago. 1998. Disponível em: <http://portal.anvisa. gov.br/wps/wcm/connect/4d02b9004745871490c4 $\mathrm{d} 43 \mathrm{fbc} 4 \mathrm{c} 6735 /$ PORTARIA+N\%C2\%BA+3.432-1998. pdf?MOD=AJPERES $>$. Acesso em: 23 out. 2015.

CARVALHO, D. M. T. Financiamento da assistência médico-hospitalar no Brasil. Ciênc. saúde coletiva, Rio de Janeiro, v. 12, n. 4, p. 879-892, 2007.

DAIN, S. Os vários mundos do financiamento da saúde no Brasil: uma tentativa de integração. Ciênc. saúde coletiva, Rio de Janeiro, v. 12, supl., 2007, p. 1851-1864.

FERRI-DE-BARROS, F.; GIBSON, J.; HOWARD, A. An argument for explicit rationing of health resources within the public-private mix in Brazil. Cad. Saúde Pública, v. 28, n. 6, 2012, p. 1211-1212.

HARDING, A.; PREKER, A. A conceptual framework for the organizational reforms of hospitals. In: PREKER, A.; HARDING, A. Innovations in health service delivery: the corporatization of public hospitals. Washington, DC: The World Bank, 2003. p. 23-78.

JAKAB, M. et al. The Introduction of Market Forces in the Public Hospital Sector: From New Public Sector
Management to Organizational Reform. Washington, DC: The World Bank, 2002.

LA FORGIA, G. M.; COUTTOLENE, B. F. Desempenho hospitalar no Brasil: em busca da excelência. São Paulo: Singular, 2009.

LIMA, S. M. L; RIVERA, F. J. U. A contratualização nos Hospitais de Ensino no Sistema Único de Saúde brasileiro. Ciênc. saúde coletiva, Rio de Janeiro, v. 17, n. 9, p. 2507-2521, 2012.

MACHADO, C. V. Novos modelos de gerência nos hospitais públicos: as experiências recentes. Physis, v. 11, n. 1, 2001, p. 105-197.

MACHADO, J. P.; MARTINS, A. C. M.; MARTINS, M. S. Avaliação da qualidade do cuidado hospitalar no Brasil: uma revisão sistemática. Cad. Saúde Pública, Rio de Janeiro, v. 29, n. 6, p. 1063-1082, 2013.

MEDICI, A. C. Sistemas de financiamento e gestão hospitalar: uma aplicação ao caso brasileiro. In: VECINA NETO, G.; MALIK, A. M. Gestão em saúde. Rio de Janeiro: Guanabara Koogan, 2011. p. 50-72.

MORICI, M. C.; BARBOSA, A. C. Q. A gestão de recursos humanos em hospitais do Sistema Único de Saúde (SUS) e sua relação ao modelo de assistência: um estudo em hospitais de Belo Horizonte, Minas Gerais. Rev. Adm. Pública, Rio de Janeiro, v. 47, n. 1, p. 205-225, 2013.

ORGANISATION FOR ECONOMIC CO-OPERATION AND DEVELOPMENT (OECD). The OECD Health Project: Private Health Insurance in OECD countries. Paris: OECD, 2004.

SANTOS, I. S. Evidência sobre o mix público-privado em países com cobertura duplicada: agravamento das iniquidades e da segmentação em sistemas nacionais de saúde. Ciênc. saúde coletiva, Rio de Janeiro, v. 16, n. 6, p. 2743-2752, 2011.

SANTOS, M. A. B; GERSCHMAN, S. As segmentações da oferta de serviços de saúde no Brasil - arranjos institucionais, credores, pagadores e provedores. Ciênc. saúde coletiva, v. 9, n. 3, p. 795-806, 2004.

SCHEFFER, M.; BAHIA, L. O financiamento de campanhas pelos planos e seguros de saúde nas eleições de 2010. Saúde em Debate, Rio de Janeiro, v. 37, n. 96, p. 96-103, 2013. 
SLOAN, F. A. et al. Hospital ownership and cost and quality of care: is there a dime's worth of difference? $J$ Health Econ., New York, n. 20, 2001, p. 1-21.

VECINA-NETO, G.; MALIK, A. M. Tendências na assistência hospitalar. Ciênc. saúde coletiva, Rio de Janeiro, v. 12, n. 4, p. 825-839, 2007.

VICTORA, C. G. et al. Condições de saúde e inovações nas políticas de saúde no Brasil: o caminho a percorrer.
The Lancet on line, 2011. Disponível em: <http://disciplinas.stoa.usp.br/pluginfile.php/269299/mod_resource/content/1/Saude\%20no\%20Brasil\%20artigo\%20 6\%20Lancet\%202011.pdf $>$. Acesso em: 23 out. 2015.

Recebido para publicação em abril de 2015

Versão final em outubro de 2015

Conflito de interesses: inexistente

Suporte financeiro: Conselho Nacional de Desenvolvimento Científico e Tecnológico (CNPq) - Processo no 302936/2013-0 